sorptionszeit des Glycins zu erklären. Die GlucoseMobilisation ist bei intravenöser Verabreichung von Aminosäuren stärker als bei oraler Gabe (4). Der Blutzuckeranstieg als Ausdruck einer Glukoneogenese nach Verabreichung der glukoplastischen Aminosäure Glycin ist jedoch durch die Versuchsergebnisse nicht ausgeschlossen.

Die beobachtete Glykämie nach gleichzeitiger Aufnahme von Alkohol und Aminosäuren und die in allen 3 Versuchsgruppen von $30 \mathrm{Min}$. nach Trinkende an praktisch gleich hohen Blutalkoholkonzentrationen sprechen gegen eine etwaige chemische Bindung zwischen Aminosäure und Alkohol $(2,3)$.

Die nach Glycin-Gabe beobachtete Glykämie senkt nicht den Spiegel der freien Fettsäuren im Plasma und vermindert somit nicht das Angebot von freien Fettsäuren an die Leber. Für eine Schutzwirkung der Aminosäure Glycin bei der Entwicklung einer alkoholinduzierten Fettleber während der Phase des $\mathrm{Al}$ koholabbaues ergeben sich weder aus dem Verhalten der Energielieferanten im Plasma noch aus der Höhe der Blutalkoholkonzentration irgendwelche Anhaltspunkte.

\title{
Literatur
}

1. Haggard, H. W. und L. A. Greenberg, J. Pharmacol. Exper. Therap. Baltimore 68, 482 (1940). - 2. WIDMark, E., Biochem. Z. 270, 297 (1934). - 3. PRoKop, L., Zschr. ärztl. Fortbild. 52, 407 (1963). - 4. Assan, R., zit. nach einem Referat über die Pariser Diabetes-Jahrestagung 1967 „Glucagon und Kohlenhydratstoffwechsel" in Aktuelle Medizin, 29, Beil. zur Münch. Med. Wschr. 109 (1967). - 5. Popper, H., Acta hepato-splenol. (Stuttg.), 8, 279 (1961). - 6. AMmoN, H. P. T. und C. J: EsTLER, Klin. Wschr. 44, 650 (1966). - 7. Frscher, H., Münch. Med. Wschr. 45, 2353 (1967). - 8. Avenarius, H. J. und W. Eger,
Mat. Med. Nordmark 19, 283 (1967). - 9. Martini, G. A. und W. Dölle, Dtsch. med. Wschr. 90, 793 (1965). - 10. Brech, W. J. und E. S. Gordon, Klin. Wschr. 45, 906 (1967). - 11. Zöllner, N. und D. Eberhagen, Untersuchungen und Bestimmungen der Lipoide im Blut, S. 339, Springer Berlin-HeidelbergNew York (1965). - 12. Elbel, H., Blutalkohol, 2. Aufl., S. 68 Thieme, Stuttgart (1956). - 13. Elmslie, R. G. und N. HarveY, Quart. J. Alcohol 28, 555 (1967). - 14. Diese Aussage wird durch weitere bisher unveröffentlichte Befunde, die am hiesigen Institut erhoben wurden, gestützt.
Dr. J. G. Gostomzyk 78 Freiburg i. Br. Albertstr. 9

\section{Dünnschichtchromatographische Untersuchungen der Faeces-Lipide Methodik und Untersuchungsbefunde bei gesunden Probanden}

\author{
Von W. ERB und E. BöHLE \\ Aus der I. Medizinischen Universitätsklinik Frankfurt|Main (Direktor: Prof. Dr. W. Siede) \\ (Eingegangen am 6. März 1968)
}

Herrn Prof. Dr. W. Siede zum 60. Geburtstag gewidmet

Es wird eine Methode zur dünnschichtchromatographischen Trennung und quantitativen Analyse der Faeceslipide beschrieben, die sich für Routinezwecke eignet. Die Untersuchungsbefunde von 18 gesunden Probanden werden mitgeteilt und mit den bisher bekannten Daten verglichen.

The investigation of faecal lipids by thin layer chromatography. Methodology, and the results from normal probands

A method is described for the separation and quantitative determination of faecal lipids by thin layer chromatography, which is suitable for routine work. The results from 18 healthy probands are reported and compared with existing data.

Die Einführung der Dünnschichtchromatographie durch Stahr (1-4) hat die Analyse von Lipidgemischen erheblich vereinfacht. Mit Hilfe dieser Methode lassen sich nicht nur Gruppentrennungen der verschiedenen Hauptlipidfraktionen, sondern auch weitergehende Differenzierungen (z. B. Auftrennung der Phospholipide, Sterinester und Fettsäuren) durchführen.
In der vorliegenden Arbeit soll über methodische Einzelheiten dünnschichtchromatographischer - Analysen von phospholipidfreien Lipidextrakten menschlicher Faeces sowie über die Zusammensetzung des Stuhlfettes gesunder Probanden berichtet werden. Systematische Untersuchungen hierüber standen bisher noch aus. 


\section{Methodik}

\section{Lipidcxtraktion}

2-Tagesstuhlportionen werden mindestens 10 Min. lang umgerührt und so lange mit physiol. Kochsalzlösung versetzt, bis eine dünnbreiige Konsistenz erreicht ist. Anschließend erfolgt die Homogenisierung der Stuhlprobe über 2 Stdn. im automatischen Rührgerät (Ultra-Turrax Fa. Janke u. Kunkel). Von der Probe werden $6 \mathrm{~g}$ abgewogen und für die Lipidextraktion nach FolCF und Mitarbeitern $(5,6)$ in der Modifikation von BöHLE und Starck (7) eingesetzt. Dabei wird die Stuhlprobe 30 Min. mit $250 \mathrm{~m} /$ Chloroform-Methanol 1:1 am Rückflußkühler extrahiert, der Extrakt filtriert und der Rückstand nochmals mit $150 \mathrm{~m} /$ Chloroform ausgeschüttelt und filtriert. Der Chloroform-MethanolExtrakt wird anschließend mit $80 \mathrm{ml}$ 0,01proz. $\mathrm{CaCl}_{2}$-Lösung geschüttelt, und nach Phasentrennung (über Nacht) die Chloroformphase gewonnen und am Rotationsverdampfer (Büchi) bis zur Trockene eingedampft. $\mathrm{Da}$ mit diesem Arbeitsgang eine vollständige Lipidextraktion nicht gewährleistet ist, wird zur Spaltung der Seifen der gesammelte Rückstand mit $5 \mathrm{ml} 25$ proz. $\mathrm{HCl}$ angesäuert, $5 \mathrm{Min}$. zum Sieden erhitzt und nach Abkühlen mit Petroläther ausgeschüttelt. Der Petrolätherextrakt wird nach Filtration ebenfalls eingedampft und mit dem ChloroformMethanol-Extrakt vereinigt.

\section{Abtrennung der Phospholipide}

Anschließend erfolgt mit Hilfe der Säulenchromatographie an Kieselgel (Mallinckrodt 100 mesh) die Abtrennung der Phospholipide von den übrigen Lipiden („Nicht-PL“). Dabei werden zuerst mit Chloroform die "Nicht-PL“, als zweite Fraktion die Phospholipide mittels Chloroform-Methanol 1:9 eluiert. Aus aliquoten Teilen beider Fraktionen lassen sich gravimetrisch die "Nicht-PL" und Phospholipide getrennt bestimmen und die Gesamt-Lipidmenge pro $24 \mathrm{Stdn}$. errechnen. Die Fraktion der „Nicht-PL“ wird anschließend dünnschichtchromatographisch weiter verarbeitet.

\section{Dünnschichtchromatographie}

Die Vorbereitung und Beschichtung der Dünnschicht-Chromatographie-Platten erfolgt in üblicher Weise mit dem Gerätesatz der Firma Desaga. Wir verwenden Kieselgel nach StahL $(2,3)$ in einer Schichtdicke von $0,25 \mathrm{~mm}$ auf Platten der Größe $40 \times$ $200 \mathrm{~mm}$.

$\mathrm{Da}$ Verunreinigungen des Kieselgels bei der dünnschichtchromatographischen Trennung mit der Fließmittelfront wandern und bei der Auswertung störende Dunkelfärbungen im Bereich der Sterinester verursachen können, werden die Platten nach SEHER (8) zunächst leer mit Chloroform entwickelt. Hierdurch gelangen die Verunreinigungen in den oberen Teil der Platte, der bei der eigentlichen Chromatographie nicht benutzt wird und zur Beschriftung dient. Durch dieses Verfahren entfällt die Reinigung des Kieselgels vor der Beschichtung, wie sie von BLANK und Mitarbeitern ( $($ ) beschrieben wurde.

Zum Auftragen der Lipidextrakte eignet sich die Sprühpistole der Firma Desaga, die unter Verwendung einer Maske mit schmalem Spalt ein gleichmäßiges Auftragen ohne Verletzung der empfindlichen Kieselgelschicht gewährleistet. Ein quantitatives Auftragen von Lipidgemischen ist auf diese Weise jedoch nicht möglich.

Als Fließmittel für die Trennung von Lipidgemischen in der qualitativen Zusammensetzung des Stuhlfettes eignet sich, wie in Vorversuchen festgestellt wurde, besonders die Mischung Hexan/ Diäthyläther/Eisessig $(80: 20: 1,5 \mathrm{v} / \mathrm{v})$ nach PIÉ und GINER (10). Wie Abbildung 1 zeigt, ist für eine optimale Trennung der einzelnen Fraktionen der Ätheranteil im Fließmittel von entscheidender Bedeutung. Durch steigende Ätherkonzentrationen im Fließmittel gelingt in zunehmendem Maße eine Trennung der freien Sterine, freien Fettsäuren und Triglyceride im mittleren Bereich der Platte, in dem bekanntlich die Bedingungen für die Auswertung am günstigsten sind $(11,12)$. Der Zusatz von Essigsäure verhindert die störende Schleppenbildung der freien Fettsäuren.

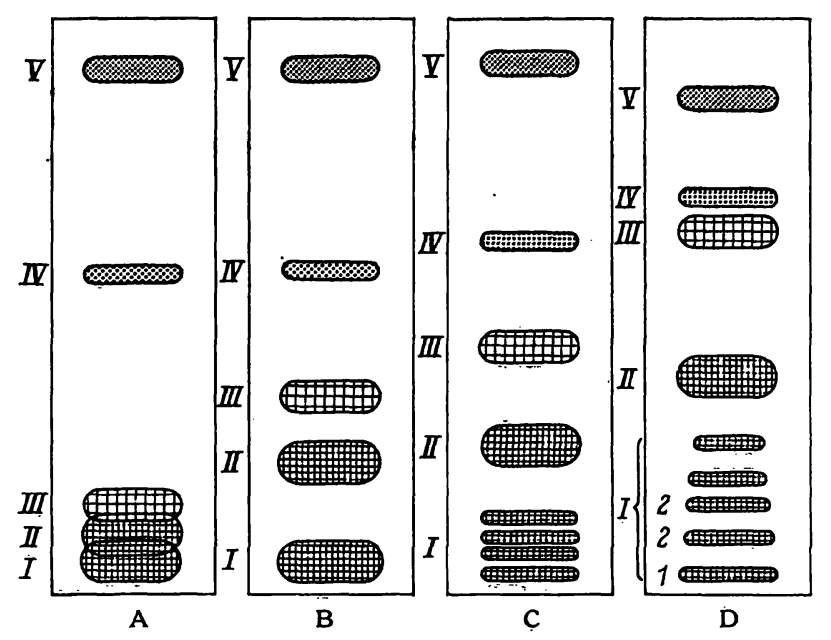

Abb. 1

Dünnschicht-Chromatogramme von Faeces-Lipidextrakten. Fließmittel: n-Hexan / Diäthyläther / Eisessig in verschiedener Zusammensetzung

Zusammensetzung des Fließmittels (v/v):
A) $90: 10: 1,5$
B) $85: 15: 1,5$
D) $70: 30: 1,5$
Fraktionen:

I Freie Sterine + (1) Mono- + (2) Diglyceride

II Freie Fettsäuren

III Triglyceride

IV Fettsäuren-Methylester

$\checkmark$ Sterinester

Die Identifizierung der Fraktionen wurde mit verschiedenen $\mathrm{Me}$ thoden vorgenommen. Durch Vergleich mit Testsubstanzen, die neben dem Lipidextrakt aufgetragen werden, lassen sich die entsprechenden Fraktionen lokalisieren. Weiterhin zeigen Sterine und ihre Ester nach Besprühen mit wäßr. Schwefelsäurelösung (etwa $2 \mathrm{~N}$ ) und anschließendem Erhitzen bei $100^{\circ}$ eine intensive rote bis violette Färbung. Schließlich können die einzelnen Fraktionen durch Besprühen mit Rhodamin B unter U.V.-Licht sichtbar gemacht und markiert werden. Dieses Verfahren eignet sich besonders zur präparativen Gewinnung und chemischen Analyse der verschiedenen Lipide.

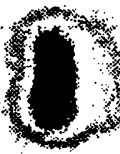

1

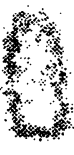

2

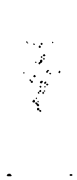

3

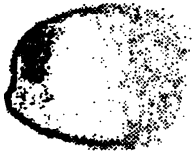

4

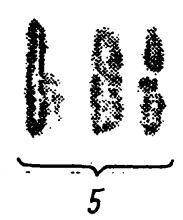

Abb. 2
Dünnśchichtchromatogramm der Faeceslipide eines Gesunden. Fließmittel Diäthyläther / n-Hexan / Eisessig (20:80:1,5 v/v) 1. Sterinester

2. Fettsäuren-Methylester

3. Triglyceride

5. freie Sterine + Diglyceride + Monoglyceride

Die Auftrennung der Faeces-Lipid-Fraktionen (Abb. 2) exfolgt nach fallenden $R_{\mathrm{F}}$-Werten in der Reihenfolge: Sterinester, Fettsäuren Methylester, Triglyceride, freie Fettsäuren, freie Sterine + Monound Diglyceride.

Die freien Sterine zeigen dabei mehrere Banden. Die stärkste Fraktion mit dem größten $R_{\text {F-Wert }}$ entspricht dem Koprosterin, die nächstfolgende dem Cholesterin. Die weiteren Fraktionen bestehen aus Steringemischen, wie gaschromatographische Analysen gezeigt haben.

Die freien Sterine kontaminieren mit Partialglyceriden (Diglyceride und Monoglyceride), deren Anteil etwa 5-10\% der Gesamtfraktion der freien Sterine ausmacht. Chemische Analysen ergaben im Bereich der freien Sterine sowohl Glycerid-Glycerin als auch Esterfettsäuren, deren Relation 1:1,62 betrug. Gegen die Annahme, daß es sich bei diesen Glyceriden um mitgeschleppte 
Triglyceride handelt, spricht nicht nur der Quotient GlyceridGlycerin:Esterfettsäuren, sondern auch die Tatsache, daß die vor den freien Sterinen wandernde Fraktion der freien Fettsäuren kein Glycerid-Glycerin entbält. Außerdem konnten die entsprechenden $R_{F}$-Werte für Mono- und Diglyceride mit Hilfe von Testsubstanzen (Monopalmitat-, Dipalmitat, Firma Serva, Heidelberg) ermittelt und im Bereich der freien Sterine nachgewiesen werden.

Bei Routineanalysen erfolgte keine Differenzierung in freie Sterine und Partialglyceride. Unter Vernachlässigung der letzteren wurde der Gesamtkomplex als „freie Sterine“ bezeichnet.

Die Lokalisation der freien Fettsäuren, der Triglyceride und der Sterinesterfraktion bereitet keine Schwierigkeit.

Problematisch erschien dagegen das Vorkommen von Fettsäuren-Methylester in den Faeces-Lipiden. Die Klärung dieser Frage gelang in der Weise, daß von mehreren Stühlen die Lipidextraktion einmal in der eingangs beschriebenen Weise, zum anderen mit Diäthyläther : Äthanol (3:1) durchgeführt wurde. Nach der dünnschicht-chromatographischen Trennung enthielt der Chloroform-Methanol-Extrakt die Fettsäuren-Methylesterfraktion, in dem Athanol-Atherextrakt war sie nicht vorhanden. Es handelt sich demnach um ein Artefakt (13). Wie durch getrennte Dünnschichtchromatographie der Extrakte 1 und 2 festgestellt werden konnte, entstehen die Fettsäuren-Methylester $z$. T. durch Methylierung beim Erhitzen des angesäuerten Rückstandes nach der ersten Extraktion. Infolgedessen ist bei der AlkoholÄther-Extraktion die Fraktion der freien Fettsäuren größer, bei der Chloroform-Methanol-Extraktion geringer. Im letzten Fall erscheint ein Teil der freien Fettsäuren als Methylester. In den übrigen Fraktionen ergaben sich bei beiden Extraktionsverfahren keine Unterschiede. Bei Routineanalysen wurden die FettsäurenMethylester den freien Fettsäuren zugerechnet.

Zur quantitativen Auswertung von Dünnschichtchromatogrammen werden verschiedene Möglichkeiten diskutiert. Neben dem Nachweisverfahren nach Wiedergewinnung der Lipidfraktionen von Dünnschichtchromatographie-Platten mittels gravimetrischer bzw. kolorimetrischer Methoden (14, 15, 16-18) oder spektrophotometrischer (19) und radiometrischer $(14,15,20-22)$ Verfahren eignen sich Methoden zur direkten quantitativen Analyse der einzelnen Fraktionen. Von Purdy und Truter $(23,24)$ sowie SEHER $(25,26)$ wurde geprüft, ob die Bestimmung der Fleckengröße der einzelnen Fraktionen eine quantitative Aussage erlaubt. Auch photoreflektorische Verfahren (27), spektrophotofluorimetrische (28) und photodensitometrische Messungen $(9,11,12)$ fanden Verwendung.

Wir haben das letztgenannte Verfahren eingehend geprüft. Hierbei wurden gut getrocknete Chromatographieplatten mit 20proz. $\mathrm{H}_{2} \mathrm{SO}_{4}$-Lösung besprüht. Anschließend erfolgte Erhitzung der Chromatogramme auf $180^{\circ}$ für $90 \mathrm{Min}$. im Brutschrank. Zur Vermeidung von Substanzverlusten durch Verdampfen sollen nach PrIVETT und Mitarbeitern $(11,12)$ höhere Temperaturen als $200^{\circ}$ nicht angewendet werden.

Die Dauer des Erhitzens ist wesentlich, damit eine vollständige Verkohlung der Lipide erreicht wird. Es hat sich gezeigt (Abb. 3), $\mathrm{da} ß$ die einzelnen Lipidfraktionen in dieser Hinsicht verschieden reagieren. Bei vergleichenden Untersuchungen von Chromatogrammen eines konstanten Testgemisches ergab sich mit steigender Ethitzungsdauer ein unterschiedlich rascher Anstieg der photometrisch erfaßbaren Größe der verschiedenen Fraktionen. Dabei erreichen die freien Fettsäuren nach mehr als einer Stunde;

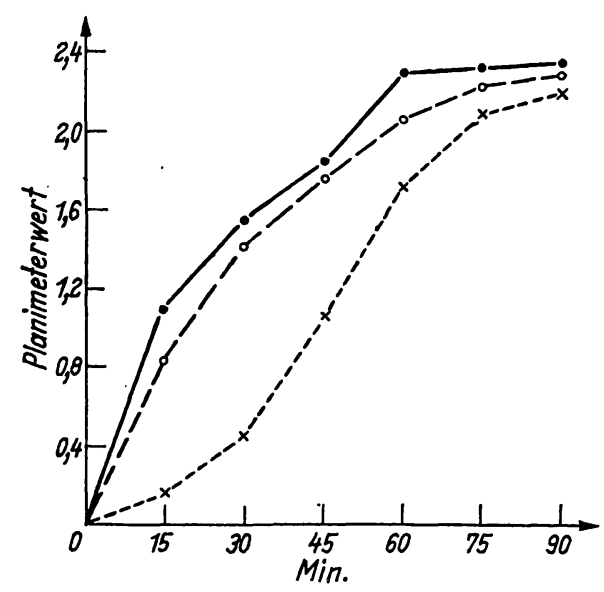

Abb. 3 Ansteigen der Planimeterwerte in Abhängigkeit von der Verkohlungs-

- Cholesterin
(Testsubstanzen, Firma SERVA)

also deutlich später als die übrigen Fraktionen, ihr Maximum, Sterine und Triglyceride verkohlten demgegenüber zeitlich schneller.

Um festzustellen, ob identische Mengen verschiedener Substanzgruppen vergleichbare Werte ergeben, wurden Gemische von Testsubstanzen : Cholerinester. Cholesterin, Triolein und Palmitin- bzw. Linolensäure (Firma Serva, Heidelberg) im Verhältnis $1: 1: 1: 1$ aufgetragen, entwickelt und ausgewertet. Die Ergebnisse in Tabelle 1 zeigen eine befriedigende Ubereinstimmung.

Weiterhin wurde zur Klärung der Frage, ob bei ansteigenden Mengen der gleichen Substanz die Auswertungsergebnisse korrespondierend ansteigen, von jeweils einer Substanz verschiedene Konzentrationen auf eine Platte $50 \times 200 \mathrm{~mm}$ aufgetragen. Diese Platte wurde in das Fließmittel gebracht, damit die Substanz bis zur Plattenmitte wandert. Die Chromatographie war nötig, weil bei alleinigem Auftragen der Substanz keine befriedigende Auswertung erzielt werden konnte. Solche Versuche wurden mit Cholesterin, Cholesterylpalmitat, Palmitinsäure und Triolein durchgeführt und Auftragsmengen im Verhältnis 3:5:7:9 gewählt. Hierbei ergab sich in allen Fällen ein fast lineares .Verhältnis von Auftragsmenge und Planimeterwert, wie es für Cholesterin in Abbildung 4 dargestellt ist.

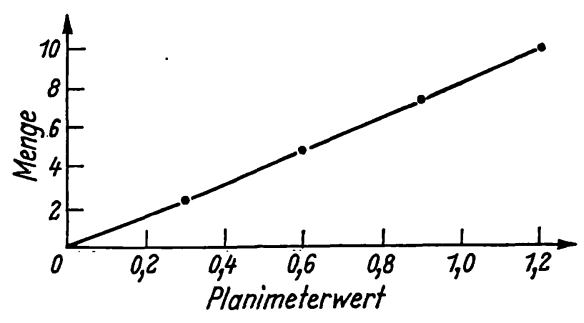

Abb. 4

Verhältnis von aufgetragener Substanzmenge zu Planimeterwert für Cholesterin. Verkohlungsdauer $90 \mathrm{Min}$. Ordinate: Menge in $\mu l$ einer 1proz. Lösung

Tab. 1

Dünnschichtchromatographische Trennung eines Testgemisches mit gleichen Anteilen der einzelnen Lipide. (Testsubstanzen der Firma SERVA.) Pl.-W. = Planimeterwert

\begin{tabular}{|c|c|c|c|c|c|c|c|c|c|c|c|}
\hline \multirow[b]{2}{*}{ Substanz } & \multicolumn{2}{|c|}{ I } & \multicolumn{2}{|c|}{ II } & \multicolumn{2}{|c|}{ Untersuchung $\mathrm{Nr}$. } & \multicolumn{2}{|c|}{ IV } & \multicolumn{2}{|c|}{ V } & \multirow{2}{*}{$\begin{array}{c}\mathrm{I}-\mathrm{V} \\
\text { Mittelwert \% }\end{array}$} \\
\hline & Pl.-W. & $\%$ & P1.-W. & $\%$ & . Pl.-W. & $\%$ & PI.-W. & $\%$ & Pl.-W. & $\%$ & \\
\hline $\begin{array}{l}\text { Cholesterin } \\
\text { Palmitinsäure } \\
\text { Cholest eryl-Palmitat } \\
\text { Triolein }\end{array}$ & $\begin{array}{l}3,3 \\
3,0 \\
3,1 \\
3,0\end{array}$ & $\begin{array}{l}26,6 \\
24,2 \\
25,0 \\
24,2\end{array}$ & $\begin{array}{l}3,0 \\
2,6 \\
3,2 \\
3,05\end{array}$ & $\begin{array}{l}25,3 \\
21,9 \\
27,0 \\
25,7\end{array}$ & $\begin{array}{l}2,0 \\
1,8 \\
2,3 \\
2,4\end{array}$ & $\begin{array}{l}23,5 \\
21,2 \\
27,1 \\
28,2\end{array}$ & $\begin{array}{l}4,3 \\
4,0 \\
4,2 \\
4,6\end{array}$ & $\begin{array}{l}25,1 \\
22,6 \\
24,6 \\
26,9\end{array}$ & $\begin{array}{l}8,4 \\
8,3 \\
8,2 \\
7,0\end{array}$ & $\begin{array}{l}26,3 \\
26,0 \\
25,7 \\
21,9\end{array}$ & $\begin{array}{l}25,3 \\
23,2 \\
25,3 \\
25,8\end{array}$ \\
\hline
\end{tabular}


Tab. 2

Faeceslipidanalysen bei 18 gesunden Probanden. Freie, gemischte Kost, 1500-2500 Kalorien

\begin{tabular}{|c|c|c|c|c|c|c|c|c|c|c|c|}
\hline \multirow[b]{2}{*}{$\begin{array}{l}\text { Mittelwert } \\
\text { Standardabweichung } \\
\text { Höchster Wert } \\
\text { Niedrigster Wert }\end{array}$} & \multirow{2}{*}{$\begin{array}{c}\begin{array}{r}\text { Stuhlfett } \\
\text { g/24 Stdn. }\end{array} \\
4,49 \\
6,20 \\
11,38 \\
1,82\end{array}$} & \multicolumn{2}{|c|}{$\begin{array}{l}\text { freie Sterine } \\
(\mathrm{g}) \quad(\%)\end{array}$} & \multicolumn{2}{|c|}{$\begin{array}{l}\text { Sterinester } \\
(\mathrm{g}) \quad(\%)\end{array}$} & \multicolumn{2}{|c|}{$\begin{array}{c}\text { Triglyceride } \\
(\mathrm{g}) \quad(\%)\end{array}$} & \multicolumn{2}{|c|}{$\begin{array}{l}\text { freie Fettsäuren } \\
\text { (g) }) \cdot(\%)\end{array}$} & \multicolumn{2}{|c|}{$\begin{array}{l}\text { Phospholipide } \\
\text { (g) }(\%)\end{array}$} \\
\hline & & $\begin{array}{l}1,89 \\
2,62 \\
3,84 \\
0,73\end{array}$ & $\begin{array}{l}42,1 \\
6,85 \\
58,8 \\
29,1\end{array}$ & $\begin{array}{l}0,42 \\
1,24 \\
1,55 \\
0,05\end{array}$ & $\begin{array}{c}9,4 \\
4,67 \\
18,9 \\
2,7\end{array}$ & $\begin{array}{l}0,40 \\
1,32 \\
1,62 \\
0,06\end{array}$ & $\begin{array}{c}8,9 \\
5,63 \\
20,5 \\
1,4\end{array}$ & $\begin{array}{l}1,50 \\
3,18 \\
3,82 \\
0,66\end{array}$ & $\begin{array}{l}33,3 \\
5,42 \\
45,0 \\
23,2\end{array}$ & $\begin{array}{l}0,28 \\
0,26 \\
0,44 \\
0,13\end{array}$ & $\begin{array}{c}6,3 \\
2,30 \\
12,1 \\
2,1\end{array}$ \\
\hline
\end{tabular}

Diese Befunde zeigten, daß das beschriebene Auswerteverfahren sowohl hinsichtlich steigender Konzentrationen der einzelnen Lipidfraktionen als auch beim Vergleich verschiedener Lipide eine hinreichende Genauigkeit bietet, sofern die Voraussetzungen einer genügend langen Verkohlungszeit erfüllt sind.

Der photometrischen Auswertung schließt sich die Planimetrie der Kurven an.

Der methodiscbe Febler wurde an Hand von 10 Doppelbestimmungen der Faeceslipide von Gesunden nach der Formel $\sqrt{\frac{\sum \Delta \mathrm{i}^{2}}{2 \mathrm{n}}}$ errechnet. Er beläuft sich auf 1,4\% für die Fraktion der freien Sterine (einschließlich Mono- und Diglyceride), auf 3,1\% für freie Fettsäuren + Fettsäuren-Methylester, 1,6\% für Triglyceride, $1,0 \%$ für Sterinester und 0,5\% für Phospholipide.

\section{Ergebnisse}

Mit der beschriebenen Methodik wurden Stuhlproben von 18 gesunden Probanden untersucht, die eine freie gemischte Kost (1500-2500 Kalorien) zu sịch nahmen.

Die Menge des 24-Stunden-Feuchtstuhles schwankte erheblich, sie lag zwischen 85 und $176 \mathrm{~g}$, im Mittel bei 122 g. Die Gesamtlipidmenge in den Faeces pro 24 Stdn. errechnete sich auf Werte zwischen 1,82 und 11,38 g bei einem Mittelwert von $4,49 \mathrm{~g}$. In den meisten Fällen (14 von 18) lag die Stuhlfettmenge unter $6 \mathrm{~g} / 24 \mathrm{Stdn}$.

Die dünnschichtchromatographischen Analysen der Faeceslipide und die Bestimmungen des Phospholipidgehaltes ergaben die in Tabelle 2 zusammengestellten Befunde.

Die prozentuale Zusammensetzung der Faeceslipidfraktionen zeigt geringe Abweichungen. Berechnet man dagegen aus den prozentualen Anteilen die Menge der pro $24 \mathrm{Stdn}$. ausgeschiedenen Lipidfraktionen, so ergeben sich infolge der stark differierenden Gesamtfettmenge im Stuhl von Fall zu Fall erheblich größere Unterschiede.

\section{Diskussion}

Die Dünnschichtchromatographie von Faeceslipiden und die quantitative Auswertung der einzelnen Fraktionen nach dem geschilderten Verfahren ergeben gut reproduzierbare Daten. Die Methode eignet sich für Routineanalysen im klinischen Labor.

Es hat sich in unseren Untersuchungen an gesunden Probanden gezeigt, daß nicht nur die Größe der fäkalen Lipidausscheidung von Mensch zu Mensch erheblich schwankt, sondern daß auch die Zusammensetzung der Stuhlfette stärkeren individuellen Variationen unterliegt. Das gilt insbesondere für die freien Sterine und freien Fettsäuren. Trotzdem ergibt die Berechnung dèr Standardabweichungen verhältnismäßig enge Grenzen bei prozentualer Auswertung der Faeces-Lipid-Fraktion. Insofern scheint das Verhältnis der einzelnen Fraktionnen zueinander relativ konstant zu sein. Demgegenüber ist die gewichtsmäßige. Verteilung der Fraktionen durch eine wesentlich größere Streubreite infolge erheblicher Unterschiede in den 24-StundenStuhlportionen ausgezeichnet. Es kann angenommen werden, daß hierbei in erster Linie die unterschiedliche Ernährung eine Rolle spielt.

Soweit vergleichbare Angaben aus der Literatur vorliegen, stimmen unsere Befunde vor allem mit den bisher bekannten Daten $(29,30,31)$ über die mit dem Stuhl ausgeschiedene Gesamtfettmenge pro $24 \mathrm{Stdn}$. gut überein. Unterschiede ergeben sich demgegenüber in der Größe der einzelnen Lipidfraktionen. Wruliams und Mitarbeiter (18) fanden mit Hilfe dünnschichtchromatographischer Untersuchungen bei Gesunden lediglich $10-15 \%$ freie Sterine und $12-20 \%$ freie Fettsäuren, daneben zwei nicht identifizierte Fraktionen in der Größenordnung von $22-32 \%$ bzw. $10-15 \%$. Es dürfte sich hierbei ebenfalls um Sterine und Fettsäuren handeln. Unter dieser Voraussetzung weichen die Ergebnisse dieser Autoren von den unserigen auch im Hinblick auf die übrigen Lipidfraktionen nur unwesentlich voneinander $\mathrm{ab}$.

Quantitative Analysen der freien Sterine im Stuhl gesunder Probanden wurden von Kinsele und Mitarbeitern (32) Mietrinen und Mitarbeitern (33) und MOore und Mitarbeitern (34) durchgeführt. Die von diesen Autoren angegebenen Tagesausscheidungen $(0,48-1,47 \mathrm{~g})$ liegen im allgemeinen unterhalb der von uns ermittelten Werte. Auch nach den Untersuchungen von BöHLE und Starck (7) ergeben sich im Vergleich zu den hier mitgeteilten Befunden niedrigere prozentuale und absolute Werte für freie Sterine. Diese Unterschiede gehen in erster Linie auf methodische Besonderheiten zurück. BÖHLE und STARCK (7) isolierten die einzelnen Faeces-Lipid-Fraktionen mittels Säulenchromatographie.

Hierbei erfolgt die Elution der Partialglyceride zusammen mit den Triglyceriden. Bei der von uns angegebenen Methode werden demgegenüber die Diglyceride und Monoglyceride mit den freien Sterinen zusammen erfaßt. Infolgedessen liegen, unsere Werte für freie Sterine höher und für Triglyceride niedriger als die von Böhle und Starck (7). Der Anteil der Phospholipide und Sterinester ist demgegenüber in beiden Fällen identisch.

Die Untersuchungen wurden mit Unterstützung der deutschen Forschungsgemeinschaft (Bo 150/10) durchgeführt. 


\title{
Literatur
}

1. Stani, E., Pharmazie 11, 633 (1956). - 2. Stark, E., Chemiker Ztg. 82, 323 (1958). - 3. Start, E., Arch. Pharmazie Berlin 292, 411 (1959). - 4. Stafi, E., Arch. Pharmazie, Berlin 293, 531 (1960). - 5. Folch, J., M. Lees und G. H. Sloans Stanley, J. Lipid Res. 1, 391 (1959). - 6. Folch, J., I. Ascoli, M. LeEs, J. A. Meath und P. N. Le Baron, J. biol. Chemistry 191, 833 (1951). - 7. BöHLE, E. und E. StarCK, Untersuchungen über die fäkale Lipidexkretion beim Menschen S. 52. Pallas Verlag München (1966). - 8. Serfer, A., Fette, Seifen einschl. Anstrichmittel 61, 345 (1959). - 9. Blank, M. I., J. A. Schmit und O. S. Privett, J. Amer. Oil Chemists' Soc. 41, 371 (1964). - 10. PrÉ, A. und A. Giner, Nature London 212, 402 (1966). - 11. Privett, O. S., M. L. Blank, D. W. Colding und E. C. Nickell, J. Amer. Oil Chemists' Soc. 42, 381 (1965). - 12. Privetr, O. S., M. L. Blank und W. LundaerG, J. Amer. Oil Chemists' Soc. 38, 297 (1961). 13. Fukuda, J., E. Mirukami und K. Imaichr, J. Biochemistry (Tokyo) 61, 657 (1967). - 14. Mangold, H. K., J. Amer. Oil Chemists' Soc. 41, 762 (1964). - 15. Mangold, H. K., J. Amer. Oil Chemists' Soc. 38, 708 (1961). - 16. Vroque, E. und L. J. Morris, J. Amer. Oil Chemists' Soc. 38, 482 (1961). - 17. VIOQUE, E., L. J. Morris und R. T. Holman, J. Amer. Oil Chemists' Soc. 38, 489 (1961): - 18. Williams, J. A., A. Sharma, L. S.
Morris und R. Holman, Proc. Soc. exp. Biol. Med. 105, 192 (1960). - 19. Gänshirt, H., F. W. Koss und W. K. Morianz, Arzneimittelforsch. Aulendorf 10, 943 (1960).-20. SNYDER, F.,Analytic. Biochem. 9, 183 (1964). - 21. SNYDER, F. und N. STEPHENS, Analytic. Biochem. 4, 128 (1962). - 22. SNYDER, F. und N. Stephens, Biochim. biophysica Acta, Amsterdam 34, 244 (1959). 23. Purdy, S. J. und E. V. Truter, Chem. and Ind. (1962), 506. - 24. Purdy, S. J. und E. V. Truter, Analyst 87, 802 (1962). - 25. SEHER, A., Mikrochim. Acta 1961, 308. - 26. Serer, A., Nahrung 4, 466 (1960). - 27. Lines, J. G., in: Biochemical problems of lipids S. 17. Hrsg. A. C. Frazer, Elsevier Publ. Co., New York (1963). - 28. Sawickr, E., T. W. Stanley und H. Thomson, Microchem. J. 8,257 (1964). - 29. BeCK, K., R. Sickinger und R. GNANK, Med. Klin. 59, 1243 (1964). - 30. Drube, H. Chr. und H. Bütrner, Klin. Wschr. 41, 749 (1963). 31. Kamer, van de, J. H., H. A. ten Bokkel Huinink und $\mathrm{H}$. A. Weyers, J. biol. Chemistry 177, 347 (1949). - 32. Krnsell, L. W., R. W. Friskey, G. D. Michaels und S. Splitter, Lancet London 1958/I 334. - 33. Mietrinen, T. A., E. H. Ahrens und S. W. GRUNDY, J. Lipid Res. 6, 411. (1965) - 34. Moore, R. B., J. T. Anderson, A. Keys und J. D. Frantz, J. Laborat Clin. Med. S. Louis 60, 1000 (1962).
Priv.-Doz. Dr. E. Böhle 6000 Frankfurt/Main 70 Ludwig-Rehn-Str. 14

\section{Analyse der Erythrocytenporphyrine}

\author{
Von M. Doss und U. BoDE \\ Aus dem Hygiene-Institut und Medizinaluntersucbungsamt der Universität Marburg (Labn) \\ (Direktor: Prof. Dr. R. Siegert) \\ (Eingegangen am 18. März 1968)
}

Die Porphyrine von Erythrocyten des Menschen wurden nach dünnschichtchromatographischer Trennung spektrophotometrisch als Methylester analysiert.

Untersuchungen von 59 Personen ergaben einen Normalbereich des Erythrocytenprotoporphyrins von 5 bis $80 \mu \mathrm{g} / 100 \mathrm{~m} l$ Erythrocyten. Die Protoporphyrinwerte des vorliegenden Probekollektivs zeigen eine log-normale Verteilung.

\section{The analysis of the porphyrins of erytbrocytes}

The porphyrins of human erythrocytes were separated by thin layer chromatography and determined spectrophotometrically as their methyl esters.

From a study of 59 persons, the normal range of erythrocyte protoporphyrin was $5-80 \mu \mathrm{g} / \mathrm{m} l$ of erythrocytes. The protoporphyrin values of the collective samples showed a log-normal distribution.

Die Methodik, freie Porphyrine im biologischen Material in Methylester zu überführen und dưrch Dünnschichtchromatographie ạn Kieselgel $\mathrm{H}(1,2) \mathrm{zu}$. isolieren, wurde zur quantitativen Analyse der Porphyrine von Erythrocyten des Menschen angewandt.

\section{Material und Methoden}

Die Erythrocyten aus 10 oder $20 \mathrm{~m} l$ Venenblut (Abnahme mit $0,1[0,2] \mathrm{m} l$ Liquemin) hämatologisch gesunder Personen werden mit physiol. $\mathrm{NaCl}-\mathrm{Lösung}$ gewaschen, gefriergetrocknet und mit Methanol- $\mathrm{H}_{2} \mathrm{SO}_{4}$ 95:5(v/v) versetzt. Nach 12 bis $14 \mathrm{Stdn}$. bei Raumtemperatur haben sich die Tetrapyrrolmethylester gebildet, die nach Abtrennung der corpuskulären Teile (Zentrifugation bei $25000 \mathrm{~g}, 10 \mathrm{Min}$.) und Zugabe von Wasser mit Chloroform ausgeschüttelt werden. Den neutralisierten, gewaschenen und auf etwa $2 \mathrm{~m} /$ eingeengten Gesamtextrakt trägt man auf eine KieselgelH-Platte (Breite $\times$ Höhe: $40 \times 20 \mathrm{~cm}$; Schichtdicke $2 \mathrm{~mm}$ ) streifenförmig auf und entwickelt das Chromatogramm im Lösungsmittelsystem Benzol/Essigsäureäthylester/Methanol 85:13,3: $1,7(\nabla / v)$ zweimal bis zu einer Höhe von $13(15) \mathrm{cm}$. Die unter dem UV-Licht $(355 \mathrm{~nm})$ rotfluoreszierenden Zonen der Trägerschicht werden in ein Glasgerät mit eingelassener Fritte (3) gesaugt, aus dem man Protoporphyrin- und Koproporphyrinmethylester mit Chloroform eluiert. Die spektrophotometrische Messung der Porphyrine erfolgt am jeweiligen Absorptionsmaximum (SoretBande). Für die Quantifizierung der Methylester stehen millimolare Extinktionskoeffizienten zur Verfügung (4). Der Porphyringehalt der Erythrocyten wurde nach folgender Formel berechnet: 